\title{
Special issue: Engineering applications of neural networks
}

\author{
Chrisina Jayne $^{1} \cdot$ Lazaros Iliadis $^{2}$
}

(C) The Natural Computing Applications Forum 2017

This is a special issue of the Neural Computing and Applications (NCA) Journal, covering the topic "Engineering Applications of Neural Networks". Artificial Neural Networks (ANNs) research and its applications have grown tremendously in the last decade due to the availability of computational power, labelled data and advances in learning algorithms. Researchers can successfully train now ANNs with many layers and solve real engineering problems.

The Engineering Applications of Neural Networks (EANN) conferences are organised annually, and the International Neural Network Society (INNS) technically supports them. Several accepted contributions were selected from the 17th EANN 2016 conference (held in Aberdeen) for potential inclusion in this special issue. Extended versions of these papers have passed through a peer review process by independent academic referees.

The accepted papers explore diverse modelling approaches and computational intelligence techniques including Multilayer Perceptron, Recurrent Neural Networks, Deep Convolutional Neural Networks, Imitation learning, Fuzzy Logic and Support Vector Machines. A number of novel methods and approaches are proposed and applied to a wide range of real-world problems such as automated clustering analysis, object tracking, 3D

Chrisina Jayne

chrisina.jayne@gmail.com

Lazaros Iliadis

liliadis@civil.duth.gr

1 School of Computing and Digital Media, Robert Gordon University, Aberdeen, UK

2 School of Engineering, Democritus University of Thrace, Xanthi, Greece navigation, stock market prediction, air pollution prediction and emotion recognition.

More specifically, in the paper "Constructive Lower Bounds on Model Complexity of Shallow Perceptron Networks", Kurkova investigates the limitations of shallow perceptron networks, with respect to computing multivariable functions on finite domains.

Ahmad and Starkey propose a novel feature weighting method in their paper "Weighted SOM approach. Application of Feature Selection Methods for Automated Clustering Analysis". The proposed method is based on the analysis of the SOM after training, allowing them to converge before analysing the input relevance. The results show that this algorithm is more effective in feature selection for clustering analysis than the weighted or standard SOM.

The paper by Masala et al. proposes a novel approach based on Recurrent Neural Networks for robust visual tracking of arbitrary objects. Its effectiveness is demonstrated on dynamic video sequences, derived from the final of triple jump at the London 2012 Summer Olympics. This algorithm could be utilised in applications for automated analysis of complex video streams.

The paper "Combining Bag-of-Words and Sentiment Features of Annual Reports to Predict Abnormal Stock Returns" authored by Hajek considers combination of financial indicators, readability, sentiment categories and bag-of-words for stock market prediction. The proposed approach performs similarly as Naïve Bayes and outperforms machine learning algorithms (Support Vector Machine, C4.5 decision tree and k-nearest neighbour classifier) in predicting positive/negative abnormal stock returns in terms of ROC.

Ruiz-Garcia et al. have authored the paper "A Hybrid Deep Learning Neural Approach for Emotion Recognition 
from Facial Expressions for Socially Assistive Robots". Their contribution investigates a new model for real-time emotion recognition, performed by a humanoid robot. This hybrid approach utilises a Deep Convolutional Neural Network for self-learnt feature extraction and a Support Vector Machine for emotion classification. Comparisons to more complex approaches with more layers in the convolutional model show that this hybrid deep learning model produces state-of-the-art classification rate on the publically available Karolinska Directed Emotional Faces dataset.

Bougoudis et al. propose a novel Hybrid Fuzzy SemiSupervised Forecasting Framework (FuSSFFra) which combines Fuzzy Logic, Semi-Supervised Clustering and Semi-Supervised Classification. This model is applied to modelling the air quality of Athens city and to predict extreme air pollutants' values.
The paper authored by Hussein et al. considers an innovative deep imitation learning method to learn navigation tasks from demonstrations in a 3D environment. The proposed approach is compared to two popular deep reinforcement learning techniques: Deep-Q-networks (DQN) and Asynchronous Actor Critic (A3C). The results show that the proposed approach can successfully learn navigation tasks from raw visual input, while learning from experience methods fails to learn an effective policy.

We hope that the readers will find these papers interesting and useful. The Guest editors wish to thank all the authors and reviewers that contributed to this special issue, and to the Editor-in-Chief and editorial office of the Neural Computing and Application journal for their support. 